\title{
Exponential polynomials in the oscillation theory
}

\author{
J. Heittokangas, K. Ishizaki, I. Laine, K. Tohge
}

\begin{abstract}
Supposing that $A(z)$ is an exponential polynomial of the form

$$
A(z)=H_{0}(z)+H_{1}(z) e^{\zeta_{1} z^{n}}+\cdots+H_{m}(z) e^{\zeta_{m} z^{n}},
$$

where $H_{j}$ 's are entire and of order $<n$, it is demonstrated that the function $H_{0}(z)$ and the geometric location of the leading coefficients $\zeta_{1}, \ldots, \zeta_{m}$ play a key role in the oscillation of solutions of the differential equation $f^{\prime \prime}+A(z) f=0$. The key tools consist of value distribution properties of exponential polynomials, and elementary properties of the Phragmén-Lindelöf indicator function. In addition to results in the whole complex plane, results on sectorial oscillation are proved.
\end{abstract}

Key Words: Exponent of convergence, exponential polynomial, differential equation, oscillation theory, Phragmén-Lindelöf indicator.

2010 MSC: Primary 34M10; Secondary 34M03.

\section{Introduction}

In the 1982 paper [1] due to Bank and Laine a sequence of results is obtained on the oscillation of solutions of the differential equation

$$
f^{\prime \prime}+A(z) f=0
$$

where $A(z)$ is an entire function. This paper prompted an extensive amount of investigations over the next three decades, not the least due to what since then has been called the Bank-Laine conjecture: If $A(z)$ is transcendental

Financially supported by the Academy of Finland \#268009, the JSPS KAKENHI Grant Numbers JP25400131 and JP16K05194, and the Discretionary Budget (2017) of the President of the Open University of Japan. 
entire with order $\rho(A) \notin \mathbb{N}$, then $\max \left\{\lambda\left(f_{1}\right), \lambda\left(f_{2}\right)\right\}=\infty$ for linearly independent solutions $f_{1}, f_{2}$ of (1.1). Here $\lambda(g)$ stands for the exponent of convergence of zeros of $g$.

Recently, the Bank-Laine conjecture has been disproved by Bergweiler and Eremenko [4, 5]. The construction of the coefficient $A(z)$ in [4] is really sophisticated, but relies on exponential polynomials of the form $P_{m}\left(e^{z}\right)$, where $P_{m}(w)$ is the $2 m$ th degree Maclaurin polynomial of the function $e^{-w}$.

Theorem A ([2]) Suppose that $A(z)=e^{z}-K$, where $K \in \mathbb{C}$. If (1.1) possesses a nontrivial solution $f$ with exponent of convergence $\lambda(f)<\infty$, then $K=q^{2} / 16$, where $q$ is an odd positive integer. Conversely, if $K$ is as above, then (1.1) with $A(z)=e^{z}-K$ has two linearly independent solutions $f_{1}, f_{2}$ for which $\lambda\left(f_{1}\right)=0=\lambda\left(f_{2}\right)$ if $q=1$ and $\lambda\left(f_{1}\right)=1=\lambda\left(f_{2}\right)$ otherwise.

Example 1 Regarding Theorem $\underline{\mathrm{A}}$, for $j=1,2$, the functions

$$
\begin{aligned}
& f_{j}(z)=\exp \left((-1)^{j} 2 i \exp \left(\frac{z}{2}\right)-\frac{z}{4}\right) \\
& f_{j}(z)=\left((-1)^{j} \frac{i}{2} \exp \left(-\frac{z}{2}\right)+1\right) \exp \left((-1)^{j} 2 i \exp \left(\frac{z}{2}\right)-\frac{z}{4}\right)
\end{aligned}
$$

are linearly independent solutions of (1.1) in the cases $q=1$ and $q=3$, respectively. The coefficient $A(z)=e^{z}-q^{2} / 16$ is clearly $2 \pi i$-periodic, which gives raise to $f_{1}(z)=i f_{2}(z+2 \pi i)$ in both cases.

The following generalization of Theorem A is commonly known as the " $\frac{1}{16}$ theorem". An improvement of this classical result will be given in Theorem 10 below.

Theorem B ([3]) Suppose that $A(z)=e^{P(z)}+Q(z)$, where $P(z)$ is a polynomial of degree $n \geq 1$ and $Q(z)$ is an entire function of order $\rho(Q)<n$. If (1.1) possesses a nontrivial solution $f$ with exponent of convergence $\lambda(f)<n$, then $f$ has no zeros and $Q(z)$ reduces to a polynomial of the form

$$
Q(z)=-\frac{1}{16} P^{\prime}(z)^{2}+\frac{1}{4} P^{\prime \prime}(z) .
$$

Moreover, (1.1) admits in this case a zero-free solution base.

This development raised further interest in the case when the coefficient $A(z)$ contains more than one exponential term.

Theorem C ([14, 15]) Suppose that $A(z)=e^{P_{1}(z)}+e^{P_{2}(z)}+Q(z)$, where $P_{1}(z)=\zeta_{1} z^{n}+\cdots$ and $P_{2}(z)=\zeta_{2} z^{m}+\cdots$ are nonconstant polynomials such that $e^{P_{1}(z)}$ and $e^{P_{2}(z)}$ are linearly independent, and $Q(z)$ is an entire function of order $<\max \{n, m\}$. Let $f$ be a nontrivial solution of (1.1). If $n \neq m$, then $\lambda(f)=\infty$, while if $n=m$, we have the following cases: 
(a) If $\zeta_{1}=\zeta_{2}$, then $\lambda(f) \geq n$.

(b) If $\zeta_{1} \neq \zeta_{2}$ and $\zeta_{1} / \zeta_{2}$ is non-real, then $\lambda(f)=\infty$.

(c) If $0<\zeta_{1} / \zeta_{2}<1 / 2$, then $\lambda(f) \geq n$.

(d) If $3 / 4<\zeta_{1} / \zeta_{2}<1$ and $Q(z) \equiv 0$, then $\lambda(f) \geq n$.

The assumption $0<\zeta_{1} / \zeta_{2}<1$ means geometrically that the points $\zeta_{1}, \zeta_{2}$ are on the same half-line emanating from the origin. In the case that $0, \zeta_{1}, \zeta_{2}$ are collinear but $\zeta_{1}, \zeta_{2}$ are on the opposite sides of the origin, we will show in Corollary 3, Section 2, that $\lambda(f)=\infty$.

As for the constants $1 / 2$ and $3 / 4$ in Theorem $[$, there are examples of zero-free solutions in the following two cases:

(1) $\zeta_{1} / \zeta_{2}=1 / 2$

(2) $\zeta_{1} / \zeta_{2}=3 / 4$ and $Q(z) \not \equiv 0$.

See [1] for (1) with $Q(z) \equiv 0$ and [14] for (2). Examples 3, 5] and 6] below deal with (1) in the case when $Q(z) \not \equiv 0$.

In this research we assume that $A(z)$ is a general exponential polynomial of the form

$$
A(z)=P_{1}(z) e^{Q_{1}(z)}+\cdots+P_{k}(z) e^{Q_{k}(z)}
$$

where $P_{j}$ 's and $Q_{j}$ 's are polynomials in $z$, and obtain several results on the oscillation of solutions of (1.1). Following Steinmetz [21], the exponential polynomial $A(z)$ in (1.2) can be written in the normalized form

$$
A(z)=H_{0}(z)+H_{1}(z) e^{\zeta_{1} z^{n}}+\cdots+H_{m}(z) e^{\zeta_{m} z^{n}}
$$

where $H_{j}(z)$ 's are either exponential polynomials of order $<n$ or ordinary polynomials in $z$, the leading coefficients $\zeta_{j}$ are pairwise distinct, and $m \leq k$.

In the further sections we pursue in the spirit of Theorem $\mathrm{C}$. For example, we show that arbitrarily many new exponential terms may be added to the exponential polynomial coefficient $A(z)$ without violating the conclusion in Theorem C(d), for as long as certain geometry of the leading coefficients does not change. Parallel discussions are already available in [24], where the approach is different from ours.

The proofs in this paper rely on properties of exponential polynomials [21] and of the Phragmen-Lindelöf indicator function [17]. These properties will be reviewed in Section 2 . 


\section{Tools and elementary observations}

We assume the fundamental results and standard notation in Nevanlinna theory [10, 16, 25]. In addition, the convex hull of a finite set $W \subset \mathbb{C}$, denoted by $\operatorname{co}(W)$, is the intersection of finitely many closed half-planes each including $W$, and hence $\operatorname{co}(W)$ is either a compact polygon or a line segment. We denote the circumference of $\operatorname{co}(W)$ by $C(\operatorname{co}(W))$. Concerning the exponential polynomial $A(z)$ in (1.3), we denote $W=\left\{\bar{\zeta}_{1}, \ldots, \bar{\zeta}_{m}\right\}$ and $W_{0}=W \cup\{0\}$.

Theorem D ([21]) Let $A(z)$ be given by (1.3) $)$. Then

$$
T(r, A)=C\left(\operatorname{co}\left(W_{0}\right)\right) \frac{r^{n}}{2 \pi}+o\left(r^{n}\right) .
$$

If $H_{0}(z) \not \equiv 0$, then

$$
m\left(r, \frac{1}{A}\right)=o\left(r^{n}\right)
$$

while if $H_{0}(z) \equiv 0$, then

$$
N\left(r, \frac{1}{A}\right)=C(\operatorname{co}(W)) \frac{r^{n}}{2 \pi}+o\left(r^{n}\right) .
$$

If $H_{0}(z) \not \equiv 0$ is a polynomial, then (2.2) can be replaced with

$$
m\left(r, \frac{1}{A}\right)=O(\log r)
$$

The Phragmén-Lindelöf indicator [17] of an entire function $f$ of order $\rho>0$ is given by

$$
h_{f}(\theta)=\limsup _{r \rightarrow \infty} r^{-\rho} \log \left|f\left(r e^{i \theta}\right)\right| .
$$

For example, if $f(z)=\exp \left(w z^{\rho}\right)$, where $w \in \mathbb{C} \backslash\{0\}$ and $\rho$ is a positive integer, then $h_{f}(\theta)=\Re\left(w e^{i \rho \theta}\right)$. It is clear that $h_{f}$ is $2 \pi$-periodic in general. If $h_{f}$ is bounded, then it is continuous. For a product and a sum of entire functions, we have $h_{f_{1} f_{2}}(\theta) \leq h_{f_{1}}(\theta)+h_{f_{2}}(\theta)$ and $h_{f_{1}+f_{2}}(\theta) \leq \max \left\{h_{f_{1}}(\theta), h_{f_{2}}(\theta)\right\}$. Moreover, if $h_{f_{1}}(\theta) \neq h_{f_{2}}(\theta)$ for some $\theta$, then

$$
h_{f_{1}+f_{2}}(\theta)=\max \left\{h_{f_{1}}(\theta), h_{f_{2}}(\theta)\right\} .
$$

Suppose that $A(z)$ is given by (1.3). Since $A(z)$ is of bounded type [21, Satz 1], its indicator $h_{A}(\theta)$ is continuous. By the proof of [21, Lemma 3], the indicator of precisely one exponential term $H_{j}(z) e^{\zeta_{j} z^{n}}$ at the time dominates the indicators of the other exponential terms in $A(z)$, except possibly for 
finitely many angles $\theta \in[-\pi, \pi)$. Thus, by (2.6) and continuity, we deduce that

$$
h_{A}(\theta)=\max _{j \leq m}\left\{\Re\left(\zeta_{j} e^{i n \theta}\right)\right\},
$$

where $\zeta_{0}=0$ if $H_{0}(z) \not \equiv 0$ and $j \geq 1$ otherwise.

Recall from [21] that if $f$ is an exponential polynomial of order $n$, then "limsup" in (2.5) can be replaced with "lim", that is,

$$
h_{f}(\theta)=\lim _{r \rightarrow \infty} r^{-n} \log \left|f\left(r e^{i \theta}\right)\right|
$$

holds for all except possibly for finitely many values of $\theta$ on $[-\pi, \pi)$. Hence for every $\theta \in[-\pi, \pi)$ with at most finitely many exceptions, we have

$$
\log \left|f\left(r e^{i \theta}\right)\right|=\left(h_{f}(\theta)+o(1)\right) r^{n}, \quad r \rightarrow \infty .
$$

This is a key property for showing that exponential polynomials are of completely regular growth, see [11, Lemma 1.3].

For an entire function $f$ of finite order and of bounded type, we may write $f^{\prime}=\left(f^{\prime} / f\right) \cdot f$ and use [8, Corollary 1] to obtain $h_{f^{\prime}}(\theta) \leq h_{f}(\theta)$ for almost all $\theta$. By continuity, it follows that

$$
h_{f^{\prime}}(\theta) \leq h_{f}(\theta)
$$

for every $\theta$. If $f$ is meromorphic in $\mathbb{C}$ of order $\rho$ with finitely many poles, then the expression $h_{f}(\theta)$ is still well-defined for every $\theta$. Recall that the poles of a meromorphic function of finite order can be enclosed in a collection of discs commonly known as an $R$-set, see [16, Chapter 5]. Therefore, even if $f$ has infinitely many poles, the indicator $h_{f}(\theta)$ is well-defined for almost all $\theta$.

The proof of Theorem $\mathrm{C}(\mathrm{d})$ in [14] is based on the following result.

Theorem E ([15]) Let $K>4$, and let $A(z)$ be a transcendental entire function of order $\rho(A)$ such that

$$
K \bar{N}\left(r, \frac{1}{A}\right) \leq T(r, A)+S(r, A)
$$

Then every nontrivial solution $f$ of (1.1) satisfies $\lambda(f) \geq \rho(A)$.

Remark 1 (1) It is apparent that the assumption (2.9) can be replaced with

$$
\Theta(0, A):=1-\limsup _{r \rightarrow \infty} \frac{\bar{N}\left(r, \frac{1}{A}\right)}{T(r, A)}>1-\frac{1}{K} .
$$


Since the sum of ramifications $\Theta(c, g)$ over $c \in \mathbb{C}$ for any entire function $g$ is at most one by [16, Corollary 2.5.5], the function $A(z)$ cannot have completely ramified values other than possibly zero by (2.10).

(2) As a special case of the original result due to Bank and Langley, [16. Theorem 5.7] asserts the following: If $A(z)=e^{P(z)}$, where $P(z)$ is a nonconstant polynomial, then every nontrivial solution $f$ of (1.1) satisfies $\lambda(f)=\infty$. The proof is rather involved even for this special case. A slightly weaker result $\lambda(f) \geq \rho(A)$ follows trivially from Theorem $\mathrm{E}$.

We state the following obvious consequence of Theorems $\mathrm{D}$ and $\mathrm{E}$.

Corollary 1 Let $A(z)$ be an exponential polynomial of the form

$$
A(z)=H_{1}(z) e^{\zeta_{1} z^{n}}+\cdots+H_{m}(z) e^{\zeta_{m} z^{n}}
$$

where the functions $H_{j}(z)$ are either exponential polynomials of order $<n$ or ordinary polynomials in z. Suppose that

$$
C\left(\operatorname{co}\left(W_{0}\right)\right)>4 C(\operatorname{co}(W)) .
$$

Then every nontrivial solution $f$ of (1.1) satisfies $\lambda(f) \geq n$.

Remark 2 (1) Suppose that $W=\left\{\bar{\zeta}_{1}, \bar{\zeta}_{2}\right\}$ and $\zeta_{1}=\rho \zeta_{2}$ for $0<\rho<1$ as in Theorem C. Then the assumption (2.12) reads as

$$
2\left|\zeta_{2}\right|>2 \cdot 2\left(\left|\zeta_{2}\right|-\left|\zeta_{1}\right|\right)=4(1-\rho)\left|\zeta_{2}\right|,
$$

from which we get $\rho>1 / 2$. Thus Corollary 1 generalizes Theorem $\mathbb{C}(\mathrm{d})$, and the result is sharp in the sense that equality in (2.12) cannot hold [14].

(2) We may add more exponential terms of order $n$ to the coefficient $A(z)$ without affecting the assertion, provided that the conjugates of the leading coefficients of the added exponential terms belong to $\operatorname{co}(W)$. Indeed, such an "addition" has no affect on (2.12). In particular, we could replace $A(z)=e^{P_{1}(z)}+e^{P_{2}(z)}$ in Theorem $\mathbb{C}(\mathrm{d})$ with $A(z)=e^{P_{1}(z)}+\cdots+e^{P_{k}(z)}$, where $P_{j}(z)$ 's are all polynomials of the same degree $n$, and the leading coefficients of $P_{3}(z), \ldots, P_{k}(z)$ are on the interval $\left[\zeta_{1}, \zeta_{2}\right]$.

(3) If the convex hull co $(W)$ has a large circumference, then it needs to be located sufficiently far away from the origin in order for (2.12) to hold.

As a further motivation to this study we re-formulate the perturbation result [3, Theorem 3.1]. This result shows that the $H_{0}(z)$-term of the exponential polynomial $A(z)$ in (1.3) plays a role in the oscillation theory. 
Theorem F ([3]) Let $A(z)$ be an exponential polynomial of order $n$, and let $f_{1}, f_{2}$ be two linearly independent solutions of (1.1) with $\max \left\{\lambda\left(f_{1}\right), \lambda\left(f_{2}\right)\right\}<$ $n$. Then, for any entire function $B(z) \not \equiv 0$ of order $\rho(B)<n$, any two linearly independent solutions $g_{1}, g_{2}$ of the differential equation

$$
g^{\prime \prime}+(A(z)+B(z)) g=0
$$

satisfy $\max \left\{\lambda\left(g_{1}\right), \lambda\left(g_{2}\right)\right\} \geq n$.

Remark 3 Prior to Theorem $\mathrm{F}$, it was shown in [2, Corollary 1] that if $A(z)$ is an exponential polynomial of the form (1.2) with non-constant polynomials $Q_{j}(z)$, then $\max \left\{\lambda\left(f_{1}\right), \lambda\left(f_{2}\right)\right\}=\infty$ for any linearly independent solutions $f_{1}, f_{2}$ of (1.1). In other words, for the assumption $\max \left\{\lambda\left(f_{1}\right), \lambda\left(f_{2}\right)\right\}<n$ in Theorem $\mathrm{F}$ to be possible, at least one of the polynomials $Q_{j}(z)$ must be constant.

The situation in the previous remark may happen. In fact, as a straight forward consequence of [16, Theorem 5.6], we next state that (1.1) may have a zero-free solution base, although this is not very typical:

Corollary 2 ([1, 16]) Let $A(z)$ be an exponential polynomial. Then (1.1) has a zero-free solution base if and only if $A(z)$ can be represented in the form

$$
-4 A(z)=e^{2 P(z)}+P^{\prime}(z)^{2}-2 P^{\prime \prime}(z),
$$

where $P(z)$ is some nonconstant polynomial. In particular, $A(z)$ in (2.13) has only one exponential term.

Theorem $\mathrm{C}$ does not address the case where $n=m$ and the points $0, \zeta_{1}, \zeta_{2}$ are collinear such that $\zeta_{1}, \zeta_{2}$ are on the opposite sides of the origin. Without loss of generality, we may assume that $\zeta_{1}>0$ and $\zeta_{2}<0$. This divides the complex plane into $2 n$ sectors in such a way that in every sector precisely one of the indicators for $e^{\zeta_{1} z^{n}}$ and $e^{\zeta_{2} z^{n}}$ is positive and the other one is negative. On the boundaries of these sectors both indicators vanish. Therefore the following result is an immediate consequence of [3, Theorem 4.3].

Corollary 3 Suppose that $A(z)=P_{1}(z) e^{\zeta_{1} z^{n}}+P_{2}(z) e^{\zeta_{2} z^{n}}+Q(z)$, where $P_{1}(z), P_{2}(z) \not \equiv 0$ and $Q(z)$ are polynomials, $\zeta_{1}>0$ and $\zeta_{2}<0$. Then every nontrivial solution $f$ of (1.1) satisfies $\lambda(f)=\infty$.

If $A(z)$ has more than a pair of collinear leading coefficients on the opposite sides of the origin, then (1.1) might have zero-free solutions.

Example 2 If $A(z)=-e^{-z}-e^{-2 z}-e^{z}-e^{2 z}+2$, then $f(z)=\exp \left(e^{-z}+e^{z}\right)$ solves (1.1). If $A(z)=-e^{-z}-e^{-2 z}+4 e^{z}-4 e^{2 z}-4 e^{4 z}$, then $f(z)=$ $\exp \left(e^{-z}+e^{2 z}\right)$ solves (1.1). Here $Q(z) \equiv 2$ and $Q(z) \equiv 0$, respectively. In both cases $h_{A}( \pm \pi / 2)=0$ and $h_{A}(\theta)>0$ elsewhere on $[-\pi, \pi)$. 


\section{Estimates of Frank-Hennekemper type}

This section contains estimates for the logarithmic derivative of meromorphic functions, which will be used in the oscillation theory later on.

The proof of Theorem E in [15] is based on the following estimate originally due to Frank and Hennekemper.

Theorem G ([6]) Let $f$ be a transcendental meromorphic function, and let $k \geq 2$ be an integer. Then

$$
m\left(r, \frac{f^{\prime}}{f}\right) \leq 2 \bar{N}\left(r, \frac{1}{f}\right)+2 \bar{N}\left(r, \frac{1}{f^{(k)}}\right)+S\left(r, \frac{f^{\prime}}{f}\right) .
$$

The original proof of (3.1) is somewhat involved. A slightly simplified proof is available in [7]. In the particular case when $f$ is a solution of (1.1) and $k=2$, the estimate (3.1) reads as

$$
m\left(r, \frac{f^{\prime}}{f}\right) \leq 2 \bar{N}\left(r, \frac{1}{f}\right)+2 \bar{N}\left(r, \frac{1}{f A}\right)+S\left(r, \frac{f^{\prime}}{f}\right) .
$$

We proceed to study estimates of the above type in the case when $f$ solves (1.1) by making a direct use of (1.1). This simplifies the proof and leads to slightly different conclusions than above.

Lemma 4 Let $f$ be a nontrivial solution of (1.1), where $A(z)$ is a transcendental entire function. Then

$$
m\left(r, \frac{f^{\prime}}{f}\right) \leq \bar{N}\left(r, \frac{1}{f f^{\prime} A}\right)+S\left(r, \frac{f^{\prime}}{f}\right) .
$$

Proof. Denote $g=f^{\prime} / f$ for short. Then $-A(z)=g^{2}+g^{\prime}$ by (1.1). Define

$$
\alpha(z):=\frac{A^{\prime}(z)}{A(z)}=\frac{2 g g^{\prime}+g^{\prime \prime}}{g^{2}+g^{\prime}} .
$$

Then we may write

$$
\left(2 g^{\prime} / g-\alpha(z)\right) g^{2}+\left(g^{\prime \prime} / g-\alpha(z) g^{\prime} / g\right) g=0
$$

If

$$
2 g^{\prime} / g-\alpha(z)=\frac{2\left(g^{\prime}\right)^{2}-g g^{\prime \prime}}{g\left(g^{2}+g^{\prime}\right)}
$$

vanishes identically, then $g^{\prime \prime} / g^{\prime}=2 g^{\prime} / g$. After integration, this transforms into a Riccati equation $g^{\prime}=C_{1} g^{2}$ for some constant $C_{1} \neq 0$. Substituting 
$g=1 / u$ into this equation, we get $u^{\prime}=-C_{1}$, and finally $g(z)=\left(C_{2}-C_{1} z\right)^{-1}$, where $C_{2} \in \mathbb{C}$. Now $f$ is either a polynomial or non-entire. Since both cases are impossible, we must have $2 g^{\prime} / g-\alpha(z) \not \equiv 0$. On the other hand, if $g^{\prime \prime} / g-\alpha(z) g^{\prime} / g \equiv 0$, then once again $g^{\prime \prime} / g^{\prime}=2 g^{\prime} / g$, which is impossible. Hence (3.4) together with the first main theorem yields

$$
\begin{aligned}
m(r, g) & \leq m\left(r, \frac{1}{2 g^{\prime} / g-\alpha}\right)+S(r, g) \\
& \leq T\left(r, 2 \frac{g^{\prime}}{g}-\alpha\right)+S(r, g) \\
& =N\left(r, 2 \frac{g^{\prime}}{g}-\frac{A^{\prime}}{A}\right)+S(r, g) .
\end{aligned}
$$

All poles of the function $G=2 g^{\prime} / g-A^{\prime} / A$ must be simple since $G$ can be expressed as the logarithmic derivative of $g^{2} / A$. Since $f$ is entire, all poles of $G$ must occur at the zeros of $f f^{\prime} A$. The assertion now follows from (3.5).

We take this opportunity to state an alternative formulation of Lemma 4, which is particularly applicable in the case when $A(z)$ is an exponential polynomial of the form (1.3).

Lemma 5 Let $f$ be a nontrivial solution of (1.1), where $A(z)=B(z)+C(z)$ is such that $A(z)$ and $B(z)$ are transcendental entire and $C(z) \not \equiv 0$ is entire. Then

$$
m\left(r, \frac{f^{\prime}}{f}\right) \leq \bar{N}\left(r, \frac{1}{f f^{\prime} B}\right)+m(r, C)+S(r, C)+S\left(r, \frac{f^{\prime}}{f}\right),
$$

unless $B(z)$ solves $B^{\prime}-\left(2 g^{\prime} / g\right) B=0$ or $B^{\prime}-\left(g^{\prime \prime} / g^{\prime}\right) B=0$, where $g=f^{\prime} / f$.

Proof. Let $g=f^{\prime} / f$. Now $-B(z)=g^{2}+g^{\prime}+C(z)$ yields

$$
\frac{B^{\prime}(z)}{B(z)}=\frac{2 g g^{\prime}+g^{\prime \prime}+C^{\prime}(z)}{g^{2}+g^{\prime}+C(z)}
$$

which we denote by $\beta(z)$ for short. Then we may write

$$
\left(2 g^{\prime} / g-\beta(z)\right) g^{2}+\left(g^{\prime \prime} / g-\beta(z) g^{\prime} / g\right) g+C^{\prime}(z)-\beta(z) C(z)=0 .
$$

If $f$ has a zero, then $g$ has a pole, and no entire function $B$ can solve either of $B^{\prime}-\left(2 g^{\prime} / g\right) B=0$ or $B^{\prime}-\left(g^{\prime \prime} / g^{\prime}\right) B=0$. Since these two equations are excluded in any case, the coefficients of $g^{2}$ and of $g$ in (3.7) do not vanish identically. Hence the rest of the proof of (3.6) follows that of Lemma 4 . 
To analyze Lemma 5, suppose that $C(z)$ is a small function in the sense that $m(r, C)=S(r, A)$. This happens, for example, if $A(z)$ is given by (1.3), where $H_{0}(z)=C(z)$. In general, we have

$$
T(r, A)=m(r, A)=m\left(r,\left(g+\frac{g^{\prime}}{g}\right) g\right) \leq 2 m(r, g)+S(r, g)
$$

Therefore (3.6) reduces to

$$
m\left(r, \frac{f^{\prime}}{f}\right) \leq \bar{N}\left(r, \frac{1}{f f^{\prime} B}\right)+S\left(r, \frac{f^{\prime}}{f}\right) .
$$

The advantage of this estimate as opposed to (3.3) is clear when $f$ is zero-free: The functions $f^{\prime}$ and $B$ may share the same zeros while $f^{\prime}$ and $A$ don't.

Example 3 If $A(z)=-\frac{1}{4}\left(e^{2 z}-2 e^{z}+4\right)$, then $f(z)=\exp \left(\frac{1}{2} e^{z}-z\right)$ is a zerofree solution of (1.1). We may choose $B(z)=-\frac{1}{4} e^{z}\left(e^{z}-2\right)$ and $C(z)=-1$ for the representation $A(z)=B(z)+C(z)$. Since $f^{\prime}=\frac{1}{2}\left(e^{z}-2\right) f$, we see that $f^{\prime}$ and $B(z)$ share the same zeros. In particular, the equality in (3.9) (as well as in (3.6) $)$ holds. Since $A(z)=-\frac{1}{4}\left(e^{z}-w\right)\left(e^{z}-\bar{w}\right)$, where $w=1+\sqrt{3} i$, the zeros of $A(z)$ are different from those of $f^{\prime}$.

Example 4 In the previous example, we have $g=f^{\prime} / f=\frac{1}{2}\left(e^{z}-2\right)$. The two special cases $B^{\prime} / B=2 g^{\prime} / g$ and $B^{\prime} / B=g^{\prime \prime} / g^{\prime}$ appearing in Lemma 5 can be obtained by choosing $B(z)=-g^{2}$ and $C(z)=-g^{\prime}$, or $B(z)=-g^{\prime}$ and $C(z)=-g^{2}$, respectively.

Remark 4 Suppose that a non-trivial solution $f$ of (1.1) has no zeros, and that $g=f^{\prime} / f$ is an exponential polynomial. Then $g^{2}+g^{\prime}$ is also an exponential polynomial, and the convex hull of its leading coefficients matches with that of $g^{2}$. Therefore

$$
2 m(r, g)=T\left(r, g^{2}\right)=T\left(r, g^{2}+g^{\prime}\right)+S(r, g)=T(r, A)+S(r, g) .
$$

Since $A(z)$ is entire, the second main theorem [10, Theorem 2.5] yields

$$
m\left(r, \frac{f^{\prime}}{f}\right) \leq 2^{-1} \bar{N}\left(r, \frac{1}{A-a_{1}}\right)+2^{-1} \bar{N}\left(r, \frac{1}{A-a_{2}}\right)+S\left(r, \frac{f^{\prime}}{f}\right)
$$

for any two distinct small target functions $a_{1}(z), a_{2}(z)$ of $A(z)$. 
Example 5 If $A(z)=-\frac{1}{16}\left(e^{z}+1\right)^{2}$, then $f(z)=\exp \left(\frac{1}{4}\left(e^{z}-z\right)\right)$ solves (1.1). Choosing $a_{1}=0$ and $a_{2}=-1 / 16$, we deduce by Theorem $\mathrm{D}$ that

$$
\begin{aligned}
m\left(r, \frac{f^{\prime}}{f}\right) & =m\left(r,\left(e^{z}-1\right) / 4\right)=\frac{r}{\pi}+O(1) \\
2 \bar{N}\left(r, \frac{1}{A}\right) & =N\left(r, \frac{1}{A}\right)=\frac{2 r}{\pi}+o(r) \\
\bar{N}\left(r, \frac{1}{A+1 / 16}\right) & =N\left(r, \frac{1}{A+1 / 16}\right)=N\left(r, \frac{1}{e^{z}+2}\right)=\frac{r}{\pi}+o(r) .
\end{aligned}
$$

Hence the equality in (3.10) holds.

Remark 5 Suppose that $f=P e^{Q}$ is a non-trivial solution of (1.1), where $P, Q$ are exponential polynomials. We may suppose that $P$ has at least two terms (in which case $P$ has infinitely many zeros), for otherwise the situation reduces essentially to the one in Remark 4. Now

$$
m\left(r, \frac{f^{\prime}}{f}\right)=m\left(r, \frac{P^{\prime}}{P}+Q^{\prime}\right)=m\left(r, Q^{\prime}\right)+O(\log r) .
$$

If $g=f^{\prime} / f$, we observe that

$$
-A=g^{2}+g^{\prime}=\left(Q^{\prime}\right)^{2}+\left(2 \frac{P^{\prime}}{P}+\frac{Q^{\prime \prime}}{Q^{\prime}}\right) Q^{\prime}+\frac{P^{\prime \prime}}{P} .
$$

An easy modification of [25, Theorem 1.12] shows that

$$
m(r, A)=2 m\left(r, Q^{\prime}\right)+O(\log r)
$$

and hence

$$
m\left(r, \frac{f^{\prime}}{f}\right) \leq 2^{-1} m(r, A)+O(\log r) .
$$

Moreover, (3.10) holds again.

Example 6 For $w_{1}=-\frac{72}{49}+\frac{6}{49} \sqrt{95}$ and $w_{2}=-\frac{72}{49}-\frac{6}{49} \sqrt{95}$, let

$$
A(z)=-\frac{49}{4}-36 e^{-z}-9 e^{-2 z}=-\frac{49}{4} e^{-2 z}\left(e^{z}-w_{1}\right)\left(e^{z}-w_{2}\right) .
$$

Then (1.1) has a solution $f(z)=\left(e^{z}+1\right)\left(e^{z}+\frac{1}{2}\right) \exp \left(3 e^{-z}-\frac{11}{2} z\right)$, for which

$$
\frac{f^{\prime}(z)}{f(z)}=-\frac{14 e^{2 z}+39 e^{z}+29+6 e^{-z}}{4\left(e^{z}+1\right)\left(e^{z}+\frac{1}{2}\right)} .
$$


Thus [22, Satz 1] gives us

$$
m\left(r, \frac{f^{\prime}}{f}\right)=\frac{r}{\pi}+o(r) .
$$

We deduce by Theorem $\mathrm{D}$ that

$$
\begin{aligned}
m\left(r, \frac{1}{A}\right) & =O(\log r) \\
\bar{N}\left(r, \frac{1}{A}\right) & =N\left(r, \frac{1}{A}\right)=\frac{2 r}{\pi}+o(r) \\
\bar{N}\left(r, \frac{1}{A+49 / 4}\right) & =N\left(r, \frac{1}{e^{-z}+4}\right)=\frac{r}{\pi}+o(r),
\end{aligned}
$$

and hence the equality in (3.11) holds while (3.10) is strict.

Example 7 If $A(z)=-\frac{1}{4} e^{-2 z}\left(e^{4 z}-4 e^{3 z}+3 e^{2 z}-4 e^{z}+1\right)$, then (1.1) has a solution $f(z)=\left(e^{z}+1\right) \exp \left(-\frac{1}{2} e^{-z}-\frac{1}{2} e^{z}-\frac{z}{2}\right)$, for which $\frac{f^{\prime}(z)}{f(z)}=\frac{e^{-z}-e^{2 z}}{2\left(e^{z}+1\right)}$. Now

$$
\begin{aligned}
& m\left(r, \frac{f^{\prime}}{f}\right)=\frac{2 r}{\pi}+o(r), \\
& \bar{N}\left(r, \frac{1}{A}\right)=N\left(r, \frac{1}{A}\right)=m(r, A)=\frac{4 r}{\pi}+o(r),
\end{aligned}
$$

and hence the equality in (3.11) holds while (3.10) is strict for the choice of $a_{1}=0$ and $a_{2} \in \mathbb{C} \backslash\{0\}$.

\section{A variant of Theorem $\mathrm{E}$}

We prove a variant of Theorem E according to which every nontrivial solution of (1.1) has either a lot of zeros or a lot of critical points. The proof relies on Frank-Hennekemper type of estimates, which were discussed in the previous section. The assertion involves a general transcendental entire coefficient $A(z)$ of finite order. We will conclude this section with remarks on the case of exponential polynomial coefficient.

Theorem 6 Let $A(z)$ be a transcendental entire function of order $\rho(A)$, and let $f$ be a nontrivial solution of (1.1).

(1) If $\bar{N}\left(r, \frac{1}{A}\right)=S(r, A)$, then

$$
\limsup _{r \rightarrow \infty} \frac{\bar{N}\left(r, \frac{1}{f}\right)}{T(r, A)} \geq \frac{1}{8} .
$$

In particular, $\bar{\lambda}(f) \geq \rho(A)$. 
(2) If $\bar{N}\left(r, \frac{1}{A}\right) \neq S(r, A)$ but (2.9) holds for $K>2$, then

$$
\limsup _{r \rightarrow \infty} \frac{\bar{N}\left(r, \frac{1}{f f^{\prime}}\right)}{\bar{N}\left(r, \frac{1}{A}\right)} \geq \frac{K-2}{2}>0 .
$$

In particular, $\max \left\{\bar{\lambda}(f), \bar{\lambda}\left(f^{\prime}\right)\right\} \geq \rho(A)$.

For the proof we need the next lemma, which is elementary but may be of independent interest in some other contexts also. Recall first that the upper (linear) density of a set $F \subset(0, \infty)$ is defined by

$$
\overline{\operatorname{dens}}(F)=\limsup _{r \rightarrow \infty} \frac{1}{r} \int_{F \cap[0, r]} d t .
$$

Lemma 7 Let $f$ and $g$ be entire functions. If $\rho(f)<\rho(g)$, then there exists a set $F \subset(0, \infty)$ such that $\overline{\operatorname{dens}}(F)=1$ and

$$
T(r, f)=o(T(r, g)), \quad r \in F .
$$

Analogous assertions $N(r, 1 / f)=o(N(r, 1 / g))$ and $N(r, 1 / f)=o(T(r, g))$ hold for $r \in F$ in the cases $\lambda(f)<\lambda(g)$ and $\lambda(f)<\rho(g)$, respectively.

Proof. Choose $\varepsilon \in\left(0, \frac{\rho(g)-\rho(f)}{3}\right)$ if $\rho(g)<\infty$, or $\varepsilon \in(0,1)$ if $\rho(g)=\infty$. Then choose $\delta \in(0, \min \{\varepsilon / 2 \rho(f), 1 / 2\})$. By the assumptions, there exists a sequence $\left\{r_{n}\right\}$ of positive numbers tending to infinity such that $T\left(r_{n}, g\right) \geq$ $r_{n}^{\rho(f)+3 \varepsilon}$. Set $s_{n}=r_{n}^{1+\delta}$. Then for $r_{n} \leq r \leq s_{n}$ and $n$ large enough,

$$
\begin{aligned}
T(r, f) & \leq r^{\rho(f)+\varepsilon} \leq s_{n}^{\rho(f)+\varepsilon}=r_{n}^{(1+\delta)(\rho(f)+\varepsilon)} \\
& \leq r_{n}^{\rho(f)+2 \varepsilon}=o\left(T\left(r_{n}, g\right)\right)=o(T(r, g)) .
\end{aligned}
$$

It is easy to see that the set $F=\cup_{n}\left[r_{n}, s_{n}\right]$ satisfies $\overline{\operatorname{dens}}(F)=1$, so that (4.3) is proved. The analogous assertions are proved similarly.

Proof of Theorem [6. (1) Let $f$ be a non-trivial solution of (1.1), where the coefficient $A(z)$ satisfies $\bar{N}\left(r, \frac{1}{A}\right)=S(r, A)$, and suppose on the contrary to the assertion (4.1) that

$$
\limsup _{r \rightarrow \infty} \frac{\bar{N}\left(r, \frac{1}{f}\right)}{T(r, A)}<\frac{1}{8} .
$$

Denoting $g=f^{\prime} / f$, we deduce from (3.2) and (3.8) that

$$
m(r, g) \leq 4 \bar{N}\left(r, \frac{1}{f}\right)+S(r, g) .
$$


Using (4.4) and (3.8) in (4.5) results in a contradiction.

Suppose on the contrary to the remaining assertion in Part (1) that $\bar{\lambda}(f)<\rho(A)$. Then Lemma 7 and (3.8) yield

$$
\bar{N}\left(r, \frac{1}{f}\right)=o(T(r, A))=o(T(r, g)), \quad r \in F,
$$

where $\overline{\operatorname{dens}}(F)=1$. Again (4.5) results in a contradiction.

(2) Let $f$ be a non-trivial solution of (1.1), where the coefficient $A(z)$ satisfies $\bar{N}\left(r, \frac{1}{A}\right) \neq S(r, A)$, yet (2.9) holds for $K>2$. Suppose on the contrary to the assertion (4.2) that

$$
\limsup _{r \rightarrow \infty} \frac{\bar{N}\left(r, \frac{1}{f f^{\prime}}\right)}{\bar{N}\left(r, \frac{1}{A}\right)}<\frac{K-2}{2} .
$$

We conclude by Lemma 4 and (4.6) that

$$
\begin{aligned}
m(r, g) & \leq \bar{N}\left(r, \frac{1}{f f^{\prime}}\right)+\bar{N}\left(r, \frac{1}{A}\right)+S(r, g) \\
& \leq(M+1) \bar{N}\left(r, \frac{1}{A}\right)+S(r, g), \quad r \geq R,
\end{aligned}
$$

where $M<(K-2) / 2$ and $R>0$ are constants. Using (2.9) and (3.8) in (4.7) results in a contradiction. The remaining assertion $\max \left\{\bar{\lambda}(f), \bar{\lambda}\left(f^{\prime}\right)\right\} \geq \rho(A)$ in Part (2) follows by Lemma 7.

Remark 6 If $A(z)$ is an exponential polynomial of the normalized form (1.3)), then the condition $\bar{N}\left(r, \frac{1}{A}\right)=S(r, A)$ in Theorem $6(1)$ is possible only in the special case when $m=1$ and $H_{0}(z) \equiv 0$. Moreover, (2.9) holds if $C\left(\operatorname{co}\left(W_{0}\right)\right)>K C(\operatorname{co}(W))$ and $H_{0}(z) \equiv 0$. However, examples in Section 3 show that $\max \left\{\bar{\lambda}(f), \bar{\lambda}\left(f^{\prime}\right)\right\} \geq \rho(A)$ may hold if $A(z)$ is an exponential polynomial which does not satisfy these requirements. In such cases the use of (3.9) instead of (3.6) may result in better estimates, as in Example 3 .

\section{A generalization of Theorem $\mathrm{C}(\mathrm{c})$}

The next result is a slight generalization of Part (c) in Theorem C.

Theorem 8 Let $A(z)$ be an exponential polynomial of the normalized form

$$
A(z)=H_{0}(z)+H_{1}(z) e^{\zeta_{1} z^{n}}+\cdots+H_{m}(z) e^{\zeta_{m} z^{n}}, \quad m \geq 2,
$$


where the functions $H_{j}(z)$ are either exponential polynomials of order $<n$ or ordinary polynomials in $z$. Denote $B(z)=A(z)-H_{1}(z) e^{\zeta_{1} z^{n}}$. Suppose that $h_{A}(\theta)>2 h_{B}(\theta)$ whenever $h_{B}(\theta)>0$, and that $C\left(\operatorname{co}\left(W_{0}^{A}\right)\right)>2 C\left(\operatorname{co}\left(W_{0}^{B}\right)\right)$. Then $\lambda(f) \geq n$ for any nontrivial solution $f$ of (1.1).

We discuss the necessity of the assumptions in Theorem 8 as follows.

Example 8 Let $f(z)=\exp \left(e^{z^{n}}\right)$, and denote $C(z)=f^{\prime}(z) / f(z)=n z^{n-1} e^{z^{n}}$. Then $f$ is a zero-free solution of (1.1), where

$$
\begin{aligned}
A(z) & =-C^{\prime}(z)-C(z)^{2} \\
& =-\left(n(n-1) z^{n-2}+n^{2} z^{2(n-1)}\right) e^{z^{n}}-n^{2} z^{2(n-1)} e^{2 z^{n}} .
\end{aligned}
$$

Defining $B(z)=A(z)+C(z)^{2}=-C^{\prime}(z)$, we have $h_{A}(\theta)=2 h_{B}(\theta)$ whenever $h_{B}(\theta)>0$, and $C\left(\operatorname{co}\left(W_{0}^{A}\right)\right)=2 C\left(\operatorname{co}\left(W_{0}^{B}\right)\right)=4$.

The proof of Theorem 8 relies partially on the ideas used in [14, 15], but is also based on Steinmetz' treatment of exponential polynomials as well as on the following lemma.

Lemma 9 Let $A(z)$ be an exponential polynomial of the form (5.1). Let $f=$ $\pi e^{g}$ be a solution of (1.1) such that $\lambda(f)=\rho(\pi)<\infty$. Then $\rho(A)=\rho(g)$, and the following assertions hold.

(1) $T(r, A) \leq 2 T\left(r, g^{\prime}\right)+O(\log r)$ and $T\left(r, g^{\prime}\right) \leq T(r, A)+O(\log r)$.

(2) If $h_{A}(\theta)>0$, then $h_{A}(\theta)=2 h_{g^{\prime}}(\theta)$.

(3) If $h_{A}(\theta) \leq 0$, then $\log ^{+}\left|g^{\prime}\left(r e^{i \theta}\right)\right| \leq O\left(r^{n-1}\right)+O(\log r)$ as $r \rightarrow \infty$ for almost every such $\theta$. Conversely, if $h_{g^{\prime}}(\theta) \leq 0$, then $\log ^{+}\left|A\left(r e^{i \theta}\right)\right| \leq$ $o\left(r^{n}\right)$ as $r \rightarrow \infty$ for almost every such $\theta$.

The assertion $\rho(A)=\rho(g)$ in Lemma 9 is known - the existing proofs are typically based on Clunie's theorem. We will give an alternative short proof which can be modified to justify the assertions in Parts (1)-(3). Remark 5 above shows that if $\pi(z)$ and $g(z)$ are exponential polynomials, then the first inequality in Part (1) is in fact an equality.

Proof of Lemma 9. A substitution of $f=\pi e^{g}$ to (1.1) gives

$$
\pi^{\prime \prime}+2 g^{\prime} \pi^{\prime}+\left(g^{\prime \prime}+\left(g^{\prime}\right)^{2}+A\right) \pi=0,
$$

where $\pi$ is a canonical product formed by the zeros of $f$. By re-writing (5.2) as

$$
A=-g^{\prime \prime}-\left(g^{\prime}\right)^{2}-2 g^{\prime} \frac{\pi^{\prime}}{\pi}-\frac{\pi^{\prime \prime}}{\pi}
$$


we see by means of a standard lemma on the logarithmic derivative that $\rho(A) \leq \max \left\{\rho\left(g^{\prime}\right), \rho\left(g^{\prime \prime}\right)\right\}=\rho(g)$. Next, we write (5.2) as

$$
\left(g^{\prime}\right)^{2}=-A-g^{\prime \prime}-2 g^{\prime} \frac{\pi^{\prime}}{\pi}-\frac{\pi^{\prime \prime}}{\pi} .
$$

Dividing (5.4) by $g^{\prime}$, and considering separately the cases $\left|g^{\prime}(z)\right| \leq 1$ and $\left|g^{\prime}(z)\right|>1$, it follows that

$$
\begin{aligned}
\log ^{+}\left|g^{\prime}(z)\right| \leq & \log ^{+}|A(z)|+\log ^{+}\left|\frac{g^{\prime \prime}(z)}{g^{\prime}(z)}\right| \\
& +\log ^{+}\left|\frac{\pi^{\prime}(z)}{\pi(z)}\right|+\log ^{+}\left|\frac{\pi^{\prime \prime}(z)}{\pi(z)}\right|+O(1)
\end{aligned}
$$

holds for every $z \in \mathbb{C}$. Therefore $\rho(g)=\rho\left(g^{\prime}\right) \leq \rho(A)$.

(1) Equation (5.3) yields

$$
\begin{aligned}
T(r, A) & =m(r, A)=m\left(r, g^{\prime}\left(\frac{g^{\prime \prime}}{g^{\prime}}+g^{\prime}+2 \frac{\pi^{\prime}}{\pi}\right)\right)+O(\log r) \\
& \leq 2 m\left(r, g^{\prime}\right)+O(\log r)=2 T\left(r, g^{\prime}\right)+O(\log r)
\end{aligned}
$$

The second assertion $T\left(r, g^{\prime}\right) \leq T(r, A)+O(\log r)$ follows similarly from (5.4).

(2) By applying [8, Corollary 1] to (5.3), we have

$$
h_{A}(\theta) \leq \max \left\{0, h_{g^{\prime \prime}}(\theta), h_{\left(g^{\prime}\right)^{2}}(\theta), h_{g^{\prime}}(\theta)\right\}
$$

for almost all $\theta$. Using (2.8) and the continuity of indicator functions, we have $h_{A}(\theta) \leq \max \left\{0, h_{\left(g^{\prime}\right)^{2}}(\theta), h_{g^{\prime}}(\theta)\right\}$ for all $\theta$. If $h_{A}(\theta)>0$, then it follows that $h_{A}(\theta) \leq 2 h_{g^{\prime}}(\theta)$. Similarly, by applying [8, Corollary 1] to (5.4), we get $2 h_{g^{\prime}}(\theta) \leq h_{A}(\theta)$ for all $\theta$ for which $h_{A}(\theta)>0$.

(3) Let $\theta$ be such that $h_{A}(\theta) \leq 0$. Since $A(z)$ is an exponential polynomial, we conclude that $\log ^{+}\left|A\left(r e^{i \theta}\right)\right| \leq O\left(r^{n-1}\right)+O(\log r)$. The assertion for $g^{\prime}$ follows from (5.5) by means of [8, Corollary 1]. Conversely, if $h_{g^{\prime}}(\theta) \leq 0$, then $\log ^{+}\left|g^{\prime}\left(r e^{i \theta}\right)\right| \leq o\left(r^{n}\right)$ as $r \rightarrow \infty$ by the definition of the indicator. The assertion for $A(z)$ follows from (5.3).

If $H_{0}(z) \not \equiv 0$ in (5.1), then $h_{A}(\theta) \geq 0$ for every $\theta$. It is possible that $h_{A}(\theta)=0$ on an interval or on finitely many subintervals of $[-\pi, \pi)$. A trivial example would be $A(z)=e^{z}$. The leading coefficients can also be chosen such that $h_{A}(\theta)=0$ only on finitely many points on $[-\pi, \pi)$, and $h_{A}(\theta)>0$ on the rest of the interval. For example, $A(z)=e^{z}+e^{-z}$ has this property. In such a case $h_{A}(\theta)=2 h_{g^{\prime}}(\theta)$ holds for every $\theta$ by the continuity of (bounded) indicator functions. 


\section{Example 9 If}

$$
\begin{aligned}
A(z)= & e^{i z}+e^{-i z}-e^{z}-e^{-z}+e^{2 i z}+e^{-2 i z}-e^{2 z}-e^{-2 z} \\
& +2 i e^{(1-i) z}+2 i e^{(i-1) z}-2 i e^{(1+i) z}-2 i e^{-(1+i) z}
\end{aligned}
$$

then (1.1) possesses a zero-free solution $f(z)=\exp \left(e^{z}+e^{-z}+e^{i z}+e^{-i z}\right)$. The convex hull of the conjugates of the leading coefficients of $A(z)$ is a square determined by the corners $\pm 2, \pm 2 i$ and has a circumference $8 \sqrt{2}$. In particular, (1.1) may have zero-free solutions even if $h_{A}(\theta)>0$ for every $\theta$.

Proof of Theorem 8. Suppose on the contrary to the assertion that $f=\pi e^{g}$ with $\lambda(\pi)<n$ solves (1.1). We may choose a constant $\alpha>0$ such that $\max \{\lambda(\pi), n-1\}<\alpha<n$. Substituting $f$ in (1.1) gives us (5.4). Then eliminating $e^{\zeta_{1} z^{n}}$ from (5.4) and writing $R=H_{1}^{\prime} / H_{1}+n \zeta_{1} z^{n-1}$, we have

$$
2 U g^{\prime}=C+D
$$

where

$$
\begin{aligned}
& U=g^{\prime \prime}-R g^{\prime} / 2 \\
& C=\sum_{j=2}^{m}\left(R H_{j}-H_{j}^{\prime}-n \zeta_{j} z^{n-1} H_{j}\right) \exp \left(\zeta_{j} z^{n}\right), \\
& D=-g^{\prime \prime \prime}+\left(R-2 \frac{\pi^{\prime}}{\pi}\right) g^{\prime \prime}+2\left(R \frac{\pi^{\prime}}{\pi}-\left(\frac{\pi^{\prime}}{\pi}\right)^{\prime}\right) g^{\prime}+R \frac{\pi^{\prime \prime}}{\pi}-\left(\frac{\pi^{\prime \prime}}{\pi}\right)^{\prime} .
\end{aligned}
$$

If $R H_{j}-H_{j}^{\prime}-n \zeta_{j} z^{n-1} H_{j}=0$ for some index $j$, then $H_{j}$ solves

$$
H_{j}^{\prime}+\left(n\left(\zeta_{j}-\zeta_{1}\right) z^{n-1}-\frac{H_{1}^{\prime}(z)}{H_{1}(z)}\right) H_{j}=0,
$$

that is, $H_{j}(z)=K H_{1}(z) \exp \left(\left(\zeta_{1}-\zeta_{j}\right) z^{n}\right)$ for some constant $K \in \mathbb{C}$. But this is a contradiction since $\zeta_{j} \neq \zeta_{1}, H_{j} \neq \equiv$ and $\rho\left(H_{j}\right) \leq n-1$ for all $j$.

We may write (5.6) in the alternative form

$$
F_{1} g^{\prime}=F_{2}
$$

where

$$
\begin{aligned}
& F_{1}=2 U+\frac{1}{2} R^{\prime}-\frac{1}{4} R^{2}-R \frac{\pi^{\prime}}{\pi}+2 \frac{\pi^{\prime \prime}}{\pi}-2\left(\frac{\pi^{\prime}}{\pi}\right)^{2}, \\
& F_{2}=-U^{\prime}+\frac{1}{2} R U-2 \frac{\pi^{\prime}}{\pi} U+R \frac{\pi^{\prime \prime}}{\pi}-\frac{\pi^{\prime \prime \prime}}{\pi}+\frac{\pi^{\prime \prime} \pi^{\prime}}{\pi^{2}}+C .
\end{aligned}
$$


Using the representation (5.6), we proceed to show that $F_{1} \equiv 0$ and $F_{2} \equiv 0$. A key step is to prove that

$$
T(r, U)=O\left(r^{\alpha}\right) .
$$

The possible poles of $U$ are among the zeros of $H_{1}$, thus $V=U H_{1}$ is an entire function. Equation (5.6) can now be written as

$$
2 V g^{\prime}=(C+D) H_{1} .
$$

As a passage to (5.9), we prove

$$
\log ^{+}\left|V\left(r e^{i \theta}\right)\right|=O\left(r^{\alpha}\right), \quad r \rightarrow \infty, \theta \notin E_{0},
$$

where $E_{0} \subset[-\pi, \pi)$ is of measure zero. If $H_{1}$ is a polynomial, then $R$ is a rational function, and so $\log ^{+}|R(z)|=O(\log |z|)$. Hence we suppose that $m=\rho\left(H_{1}\right) \geq 1$, and keep in mind that $m \leq n-1<\alpha$. By making use of (2.7) and (2.8) for $H_{1}$ instead of $f$, we deduce that

$$
\log ^{+}\left|R\left(r e^{i \theta}\right)\right|=o\left(r^{m}\right), \quad r \rightarrow \infty,
$$

for every $\theta \in[-\pi, \pi)$ with at most finitely many exceptions. If $z$ is such that $\left|g^{\prime}(z)\right| \leq 1$, then $U=\left(g^{\prime \prime} / g^{\prime}-R / 2\right) \cdot g^{\prime}$ together with (5.12) and [8, Corollary 1] yield

$$
\log ^{+}|V(z)| \leq \log ^{+}\left(\left|\frac{g^{\prime \prime}(z)}{g^{\prime}(z)}\right|+\frac{1}{2}|R(z)|\right)+\log ^{+}\left|H_{1}(z)\right| \leq O\left(|z|^{\alpha}\right)
$$

as $|z| \rightarrow \infty$ with $\arg (z) \notin E_{0}$, where meas $\left(E_{0}\right)=0$. Hence from now on we may suppose that $\left|g^{\prime}(z)\right|>1$. If $\theta \notin E_{0}$ is such that $h_{C}(\theta) \leq 0$, then

$$
\log ^{+}\left|C\left(r e^{i \theta}\right)\right| \leq O\left(r^{n-1}\right)
$$

and dividing (5.10) by $2 g^{\prime}$ gives us (5.11). On the other hand, if $\theta \notin E_{0}$ is such that $h_{C}(\theta)>0$, then $h_{B}(\theta)=h_{C}(\theta)>0$, since we have proved that none of the coefficients of the exponential polynomial $C$ vanishes. We have by the assumption and by Lemma 9 that

$$
2 h_{g^{\prime}}(\theta)=h_{A}(\theta)>2 h_{C}(\theta) .
$$

Dividing (5.10) again by $2 g^{\prime}$ gives us (5.11) in this case also.

Since $V$ is entire and of finite order, we may use the standard PhragménLindelöf principle to deduce that the estimate in (5.11) is uniform, and that the exceptional set $E_{0}$ can be ignored. Therefore $m(r, V)=O\left(r^{\alpha}\right)$, and so

$$
m(r, U)=m\left(r, V / H_{1}\right) \leq m(r, V)+T\left(r, H_{1}\right)+O(1)=O\left(r^{\alpha}\right) .
$$


Finally, since $\lambda(1 / U) \leq n-1$, the assertion (5.9) follows.

Next, we continue the proof by estimating $T\left(r, F_{1}\right)$ and $T\left(r, F_{2}\right)$ under the assumption that $F_{1} F_{2} \not \equiv 0$. Since $\rho(R) \leq n-1$ and $\rho(\pi)<\alpha$, we obtain by (5.9) that

$$
T\left(r, F_{1}\right)=O\left(r^{\alpha}\right) .
$$

In the same manner we deduce that

$$
T\left(r, F_{2}\right) \leq T(r, C)+O\left(r^{\alpha}\right) .
$$

The identity $T(r, C)=T(r, B)+O\left(r^{\alpha}\right)$ being clear by Theorem $\mathrm{D}$, we then get from (5.7) that

$$
T\left(r, g^{\prime}\right) \leq T\left(r, F_{1}\right)+T\left(r, F_{2}\right)+O(1) \leq T(r, B)+O\left(r^{\alpha}\right) .
$$

By using the assumption $C\left(\operatorname{co}\left(W_{0}^{A}\right)\right)>2 C\left(\operatorname{co}\left(W_{0}^{B}\right)\right)$ together with Lemma 9 and Theorem $\mathrm{D}$, we arrive at a contradiction. Hence at least one of $F_{1}$ or $F_{2}$ must vanish. A fortiori they both vanish by (5.7).

Finally, since $F_{2} \equiv 0$, we infer from (5.8) that

$$
T(r, C)=O\left(r^{\alpha}\right),
$$

which is clearly a contradiction. Thus $\lambda(f) \geq n$.

\section{An improvement of the $\frac{1}{16}$-theorem}

The following improvement of the $\frac{1}{16}$-theorem (see Theorem $\mathrm{B}$ ) is motivated by the assumptions in Theorem 8 .

Theorem 10 Suppose that $A(z)=T(z)+B(z)$, where

$$
T(z)=H(z) e^{\zeta z^{n}}, \quad \zeta \in \mathbb{C} \backslash\{0\},
$$

$H(z)$ is an entire function of order $<\alpha<n$, and $B(z)$ is a finite order entire function with the following property: For each $\theta \in[-\pi, \pi)$ there exists $s(\theta)<1 / 2$ with

$$
\log ^{+}\left|B\left(r e^{i \theta}\right)\right| \leq s(\theta) \max \left\{\Re\left(\zeta e^{i n \theta}\right), 0\right\} r^{n}+r^{\alpha},
$$

as $r \rightarrow \infty$, but not necessarily uniformly in $\theta$. If (1.1) possesses a nontrivial solution $f$ with $\lambda(f)<\alpha<n$, then $f$ and $H(z)$ have no zeros, and

$$
B(z)=-\frac{1}{16}\left(\frac{T^{\prime}(z)}{T(z)}\right)^{2}+\frac{1}{4}\left(\frac{T^{\prime}(z)}{T(z)}\right)^{\prime} .
$$

Moreover, (1.1) admits in this case a zero-free solution base. 
It is easy to see that (6.2) forces $B(z)$ to be a polynomial of degree $2(n-1)$ with leading term $-16^{-1} n^{2} \zeta^{2} z^{2(n-1)}$, so the situation is not that much different from that in Theorem B. As for the sharpness of Theorem 10, if we permit $s(\theta)$ to attain the value $1 / 2$, then the existence of a zero-free solution does not imply (6.2), see Example 8 .

Lemma 11 Let $g$ be an entire function of order $\rho(g)<\alpha$. Then $|\log | g(z)|| \leq$ $|z|^{\alpha}$ for all $z$ outside of an $R$-set.

Proof. The inequalities $\log |g(z)| \leq \log M(|z|, g) \leq|z|^{\alpha}$ being trivial, it suffices to prove $\log |g(z)| \geq-|z|^{\alpha}$ for suitable $z$. We may write $g=P e^{h}$, where $h$ is a polynomial of degree $<\alpha$, and $P$ is a canonical product formed with the zeros $z_{n}$ of $g$. If $g$ has no zeros or has at most finitely many zeros (that is, $P$ is a polynomial), the assertion is trivial. If $P$ is transcendental, then [23, Theorem V. 19] gives us

$$
\log ^{+} \frac{1}{|P(z)|}=O\left(|z|^{\rho(P)+\varepsilon}\right)
$$

for all $z$ outside of an $R$-set [16, p. 84], where we have fixed $\varepsilon<\alpha-\rho(P)$. This yields the assertion.

Proof of Theorem 10. First we observe that the assumption (6.10 together with the Phragmén-Lindelöf principle yield $T(r, B)=O\left(r^{n}\right)$. Moreover, the assumption (6.1) holds with $B$ being replaced by $B^{\prime}$ for almost all $\theta$.

Set $F(z)=f^{\prime}(z) / f(z)$. Then $\lambda(1 / F)<\alpha$ by the assumption, and so $\rho(F)<\alpha$ by the standard lemma on the logarithmic derivative. By [16, Proposition 5.12] there exists a constant $N_{0}>0$ and an $R$-set $E_{1}$ surrounding the zeros of $f$ such that

$$
\left|\frac{F^{\prime \prime}(z)}{F(z)}\right|+\left|\frac{F^{\prime}(z)}{F(z)}\right|+\left|\frac{T^{\prime}(z)}{T(z)}\right|=O\left(|z|^{N_{0}}\right), \quad z \notin E_{1} .
$$

By Lemma 11 there exists an $R$-set $E_{2}$ such that

$$
|\log | H(z)|| \leq|z|^{\alpha}, \quad z \notin E_{2} .
$$

Recall that the set of angles $\theta$ for which the ray $r e^{i \theta}$ meets infinitely many discs of a given $R$-set has linear measure zero by [16, Lemma 5.9]. Thus the estimates in (6.3) and (6.4) are valid for almost all $\theta$ as $|z| \rightarrow \infty$.

We have the identities

$$
\begin{gathered}
F^{\prime}+F^{2}=-A=-T-B, \\
F^{\prime \prime}+2 F F^{\prime}=-T^{\prime}-B^{\prime}=t\left(F^{\prime}+F^{2}+B\right)-B^{\prime},
\end{gathered}
$$


where $t=T^{\prime} / T$ has order $\rho(t)<\alpha$. Set $G=2 F^{\prime}-t F$. Then (6.6) yields

$$
F G=2 F F^{\prime}-t F^{2}=-F^{\prime \prime}+t\left(F^{\prime}+B\right)-B^{\prime} .
$$

There exists an entire function $U \not \equiv 0$ with $\rho(U)<\alpha$ such that $J=G U$ is entire, and

$$
F J=F G U=-U\left(F^{\prime \prime}-t\left(F^{\prime}+B\right)+B^{\prime}\right) .
$$

It is clear that $\rho(G)<\alpha$, so that $\rho(J)<\alpha$.

We easily obtain

$$
F^{2}+t F / 2=-T-B-K
$$

where $K=F^{\prime}-t F / 2=G / 2$, and so

$$
L^{2}=-T-B+M
$$

where $L=F+t / 4$ and $M=t^{2} / 16-K$. The functions $K, L, M$ are meromorphic and of order $\leq \alpha$. It is clear that $L \not \equiv 0$ because $f$ is of infinite order, while $T$ is of finite order. A differentiation of (6.8) gives

$$
2 L L^{\prime}=-B^{\prime}+M^{\prime}-T^{\prime}=-B^{\prime}+M^{\prime}+t\left(F^{\prime}+F^{2}+B\right),
$$

followed by a simple manipulation

$$
L\left(2 L^{\prime}-t L\right)=-B^{\prime}+M^{\prime}+t(B-M) .
$$

Set $N=2 L^{\prime}-t L$. The crux of the proof is to show that

$$
N \equiv 0 \text {. }
$$

Suppose on the contrary to this that $N \not \equiv 0$. The poles of $N$ are among the zeros of $f$ and $T$. Hence $\lambda(1 / N)<\alpha$, and so there exists an entire function $Q \not \equiv 0$ of order $\leq \alpha$ such that $S=N Q$ is entire. Moreover, (6.9) yields

$$
\begin{aligned}
L S & =L N Q=-Q\left(B^{\prime}-M^{\prime}+t(M-B)\right) \\
& =-Q\left(\left(\frac{B^{\prime}}{B}-t\right) B-M^{\prime}+t M\right) .
\end{aligned}
$$

We proceed similarly as above. First, we find that there exists a constant $N_{1}>0$ and an $R$-set $E_{3} \supset E_{1} \cup E_{2}$ surrounding the zeros of $f$ and $T$ such that

$$
\left|\frac{F^{\prime \prime}(z)}{F(z)}\right|+\left|\frac{F^{\prime}(z)}{F(z)}\right|+\left|\frac{L^{\prime}(z)}{L(z)}\right|=O\left(|z|^{N_{1}}\right), \quad z \notin E_{3},
$$


and

$$
|\log | H(z)||+\log ^{+}|M(z)|+\log ^{+}\left|M^{\prime}(z)\right| \leq|z|^{\beta}, \quad z \notin E_{3},
$$

where $\alpha<\beta<\gamma<n$. Second, we find sectorial estimates for $L$ and $S$. For almost all $\theta$ for which $h_{T}(\theta)>0$, we have

$$
\Re\left(\zeta e^{i n \theta}\right) r^{n}-O\left(r^{\alpha}\right) \leq \log \left|T\left(r e^{i \theta}\right)\right|
$$

by (6.4) and then $2 h_{L}(\theta)=h_{T}(\theta)$ by (6.8). Dividing (6.11) by $L$, we have by (6.1) and (6.12) that

$$
\begin{aligned}
\log \left|S\left(r e^{i \theta}\right)\right| & \leq(s(\theta)-1 / 2) \Re\left(\zeta e^{i n \theta}\right) r^{n}+O\left(r^{\beta}\right) \\
& \leq(s(\theta)-1 / 2) \Re\left(\zeta e^{i n \theta}\right) r^{n} / 2<0
\end{aligned}
$$

for all $r$ sufficiently large. In other words, $h_{S}(\theta)<0$ for almost all $\theta$ for which $h_{T}(\theta)>0$. Similarly, for almost all $\theta$ for which $h_{T}(\theta)<0$, we have

$$
\log ^{+}\left|L\left(r e^{i \theta}\right)\right|=O\left(r^{\beta}\right)
$$

by (6.1) and (6.8), and

$$
\begin{aligned}
\log ^{+}\left|S\left(r e^{i \theta}\right)\right| & \leq \log ^{+}\left|N\left(r e^{i \theta}\right)\right|+O\left(r^{\beta}\right) \\
& \leq \log ^{+}\left|2 \frac{L^{\prime}}{L} \cdot L\right|+\log ^{+}|t L|+O\left(r^{\beta}\right)=O\left(r^{\beta}\right) .
\end{aligned}
$$

Thus the Phragmén-Lindelöf principle again implies that $\rho(S) \leq \beta$, and hence $\rho(N) \leq \beta$. Therefore we may find an $R$-set $E_{4} \supset E_{3}$ such that

$$
|\log | S(z)|| \leq|z|^{\gamma}, \quad z \notin E_{4}
$$

But this contradicts the fact that (6.13) holds for $\theta$ in a set of positive measure.

We deduce by (6.10) that $L=c T^{1 / 2}$ for some $c \in \mathbb{C} \backslash\{0\}$. Hence

$$
F=c T^{1 / 2}-t / 4 \quad \text { and } \quad F^{\prime}=c t T^{1 / 2} / 2-t^{\prime} / 4,
$$

which together with (6.5) imply

$$
-T-B=F^{\prime}+F^{2}=c^{2} T+t^{2} / 16-t^{\prime} / 4 .
$$

This yields $c^{2}=-1$ and $B=-t^{2} / 16+t^{\prime} / 4$, which proves (6.2).

If $H$ has a zero of multiplicity $m \geq 1$ at some point $\zeta_{0}$, then $T(z)=$ $a_{m}\left(z-\zeta_{0}\right)^{m}(1+o(1))$ and

$$
\frac{T^{\prime}(z)}{T(z)}=\frac{m}{z-\zeta_{0}}(1+o(1))
$$


near $\zeta_{0}$. But then $B$ has a double pole at $\zeta_{0}$ by (6.2), which is a contradiction. Hence $H$ has no zeros. Thus $P(z)=\zeta z^{n}+\log H(z)$ is a polynomial of degree $n$. The proof of the fact that (1.1) has a zero-free solution base $\left\{f_{1}, f_{2}\right\}$ is similar to the reasoning in [3, p. 8] or [1, p. 356]. If $g$ is any linear combination of $f_{1}, f_{2}$, then $\lambda(g)=\infty$. Thus, if a nontrivial solution $f$ of (1.1) satisfies $\lambda(f)<n$, it must be a constant multiple of one of $f_{1}, f_{2}$, and hence has no zeros.

\section{Oscillation in sectors}

Instead of oscillation, Steinbart takes a step in the opposite direction by proving three results on non-oscillation in the case when the coefficient $A(z)$ is an exponential polynomial [20]. Under certain assumptions, the equation (1.1) is proven to be non-oscillatory in a sector, that is, every non-trivial solution of (1.1) has at most finitely many zeros in the sector in question. The main results in [20] are quite technical, and the proofs are very involved relying on Strodt's theory.

Assuming that the coefficient $A(z)$ is entire and sufficiently small in an unbounded quasidisc $D$, Hinkkanen and Rossi proved [13] that any nontrivial solution of (1.1) has at most one zero in $D$. As a consequence of their main result, if $A(z)=P(z) e^{Q(z)}$, where $P(z), Q(z)$ are polynomials, one can usually take $D$ to be a sector of opening $\pi / \operatorname{deg}(Q)$.

The next result is stated for an entire coefficient $A(z)$ of finite order, but the assumptions are quite typical properties of exponential polynomials. The proof is rather elementary and relies on a well known univalence criterion due to Nehari.

Theorem 12 Let $A(z)$ be an entire function of order $\rho(A)=\rho \in[1, \infty)$. Suppose that there exists two zeros $\alpha, \beta \in \mathbb{R}$ of the indicator function $h_{A}$ such that $0<\beta-\alpha \leq \pi / \rho$ and $h_{A}(\theta)<0$ for $\theta \in(\alpha, \beta)$. Then, for any $\varepsilon>0$, every nontrivial solution $f$ of (1.1) has at most finitely many zeros in the sector $\alpha+\varepsilon<\arg (z)<\beta-\varepsilon$. The number of zeros may depend on $\varepsilon$.

Remark 7 In fact, the result holds if $A(z)$ only tends to zero exponentially in some sector $S$ as in (7.1), but independently on the order of $A(z)$ or the indicator of $A(z)$. However, the authors feel that the current statement is more natural due to two reasons: (a) Functions of order $<1 / 2$ do not have this property by the $\cos \pi \rho$-theorem. (b) If an entire function $A(z)$ is of order $\rho<1$ and tends to zero in an angle of opening $\pi / \rho$, then it must vanish identically by theorems due to Phragmén-Lindelöf and Liouville. 
Proof of Theorem 12. Let $\varepsilon \in(0,(\beta-\alpha) / 2)$, and recall the fundamental relation of the indicator from [17, p. 53]:

$$
h_{A}(\theta) \leq \frac{h_{A}\left(\theta_{1}\right) \sin \rho\left(\theta_{2}-\theta\right)+h_{A}\left(\theta_{2}\right) \sin \rho\left(\theta-\theta_{1}\right)}{\sin \rho\left(\theta_{2}-\theta_{1}\right)},
$$

where $\theta_{1}<\theta<\theta_{2}$ and $0<\theta_{2}-\theta_{1}<\pi / \rho$. Choose $\theta_{1}=\alpha+\varepsilon / 2$ and $\theta_{2}=$ $\beta-\varepsilon / 2$, and note that $h_{A}\left(\theta_{1}\right)<0$ and $h_{A}\left(\theta_{2}\right)<0$. We conclude that there exists a constant $\delta_{0}>0$ such that $h_{A}(\theta)<-\delta_{0}$ for every $\theta \in(\alpha+\varepsilon, \beta-\varepsilon)$. By the Phragmén-Lindelöf theorem for an angle, there exist constants $\delta \in\left(0, \delta_{0}\right)$ and $R>0$ such that

$$
|A(z)| \leq \exp \left(-\delta|z|^{\rho}\right), \quad z \in S(\alpha+\varepsilon, \beta-\varepsilon, R),
$$

where $S(a, b, R)=\{z \in \mathbb{C}: a<\arg (z)<b,|z|>R\}$.

Next we construct a conformal mapping $\Phi$ from $\mathbb{D}$ onto $S(\alpha+\varepsilon, \beta-\varepsilon, R)$ in two parts. First we construct a conformal mapping $T$ from $\mathbb{D}$ onto a lensshaped domain $D$ symmetric with respect to the $x$-axis and bounded by an arc of $\partial \mathbb{D}$ and by a Poincaré line traveling through a point $s \in(0,1)$. An exact formulation of the mapping $T$ follows by the discussions in [18, pp. 208209]. Alternatively, we may construct $T$ as a composition of elementary transformations as follows: First we map $\mathbb{D}$ onto the right half-plane $H$ by means of $z \mapsto(1+z) /(1-z)$. Then the principal branch of the square root maps $H$ onto the sector $|\arg (z)|<\pi / 4$. We map this back into the unit disc and onto a lens shaped domain with straight angles at the points $z= \pm 1$ by means of $z \mapsto(z-1) /(z+1)$. Finally, after a suitable dilatation $z \mapsto t z$ for some $t \in(0,1)$, rotation $z \mapsto i z$, and a translation $z \mapsto z+u$ for some $u \in(0,1)$, we reach our target domain $D$. This gives us

$$
T(z)=\frac{(u+i t) \sqrt{1+z}+(u-i t) \sqrt{1-z}}{\sqrt{1+z}+\sqrt{1-z}}
$$

where $u=(1+s) / 2$ and $t=(1+\sqrt{2})(1-s) / 2$. Second, a conformal mapping $L$ from $D$ onto $S(\alpha+\varepsilon, \beta-\varepsilon, R)$ is of the form

$$
L(z)=e^{i \varphi}\left(\frac{1+z}{1-z}\right)^{\gamma}
$$

where $\gamma=(\beta-\alpha-2 \varepsilon) / \pi<(\beta-\alpha) / \pi \leq 1 / \rho \leq 1$ and $\varphi=(\beta-\alpha) / 2$. Now $\Phi=L \circ T$ is the mapping we are looking for.

The Schwarzians of $L$ and $T$ can be computed directly (by computer). Alternatively, we may rely on the discussions in [18, pp. 208-209]. We have

$$
S_{T}(z)=\frac{3}{2\left(1-z^{2}\right)^{2}} \quad \text { and } \quad S_{L}(z)=\frac{2\left(1-\gamma^{2}\right)}{\left(1-z^{2}\right)^{2}} .
$$


Note in particular that the Schwarzians are independent on the constants $u, t, \varphi$. It follows that

$$
\begin{aligned}
S_{\Phi}(z) & =S_{L}(T(z)) T^{\prime}(z)^{2}+S_{T}(z) \\
& =\frac{2\left(1-\gamma^{2}\right)}{\left(1-T(z)^{2}\right)^{2}} T^{\prime}(z)^{2}+\frac{3}{2\left(1-z^{2}\right)^{2}}
\end{aligned}
$$

where

$$
\left|T^{\prime}(z)\right|^{2}=\frac{4 t^{2}}{\left|2+2 \sqrt{1-z^{2}}\right|^{2}\left|1-z^{2}\right|} \leq \frac{1}{1-|z|^{2}}, \quad z \in \mathbb{D} .
$$

If $\left|1-z^{2}\right|<1 / 2$, we use the continuity and the construction of $T(z)$ together with $T(-i)=1$ to conclude that $\left|1-T(z)^{2}\right|>\delta>0$. Thus

$$
\left(1-|z|^{2}\right)^{2}\left|S_{\Phi}(z)\right| \leq 2\left(1-\gamma^{2}\right)\left(1-|z|^{2}\right) / \delta^{2}+3 / 2
$$

If $\left|1-z^{2}\right| \geq 1 / 2$, we use the Schwarz-Pick lemma $\left(1-|z|^{2}\right)\left|T^{\prime}(z)\right| \leq 1-|T(z)|^{2}$, and obtain

$$
\left(1-|z|^{2}\right)^{2}\left|S_{\Phi}(z)\right| \leq 2\left(1-\gamma^{2}\right)+6\left(1-|z|^{2}\right)^{2} .
$$

Hence, in all cases there exists a constant $x_{0} \in(0,1)$ such that

$$
\left(1-|z|^{2}\right)^{2}\left|S_{\Phi}(z)\right|<2, \quad x_{0}<|z|<1 .
$$

Let $\left\{f_{1}, f_{2}\right\}$ be a fundamental solution base of (1.1). Define $h=f_{1} / f_{2}$, so that $S_{h}(z)=2 A(z)$. The Schwarzian of $g=h \circ \Phi$ is given by

$$
S_{g}(z)=2 A(\Phi(z)) \Phi^{\prime}(z)^{2}+S_{\Phi}(z) .
$$

Since $\Phi$ is univalent, we have $\left|\Phi^{\prime}(z)\right|=O\left(1 /(1-|z|)^{3}\right)$. Hence, if $R>0$ is large enough, or, in other words, if $s \in(0,1)$ is close enough to one, then (7.1) yields

$$
\left(1-|z|^{2}\right)^{2}\left|S_{g}(z)\right| \leq 2, \quad x_{0}<|z|<1 .
$$

By an extension of classical Nehari's theorem [19, Corollary 1], we find that $g$ is finitely valent in $\mathbb{D}$. In other words, $h$ is finitely valent in $S(\alpha+\varepsilon, \beta-\varepsilon, R)$, that is, any solution of (1.1) has at most finitely many zeros in $S(\alpha+\varepsilon, \beta-$ $\varepsilon, R)$. Hence the assertion follows.

Example 10 If $A(z)=e^{z}+e^{2 z}+e^{3 z}$, then $h_{A}(\theta)=\cos \theta<0$ for all $\theta \in$ $(\pi / 2,3 \pi / 2)$, and hence the solutions of (1.1) have at most finitely many zeros in the (slightly squeezed) left half-plane. 
Example 11 Let $A(z)$ be a solution of a differential equation $g^{\prime \prime}+P(z) g=0$, where $P(z)=a_{n} z^{n}+\cdots+a_{0}$ is a polynomial of degree $n$. Then $\rho(A)=$ $(n+2) / 2$ by [16. Proposition 5.1]. Moreover, by Hille's method of asymptotic integration [12, Section 7.4], it is known that $A(z)$ either blows up or converges to zero exponentially in sectors determined by the critical rays $\arg (z)=\theta_{j}$, where

$$
\theta_{j}=\frac{1}{n+2}\left(2 \pi j-\arg \left(a_{n}\right)\right), \quad j=0, \ldots, n+1 .
$$

Since $\theta_{j+1}-\theta_{j}=2 \pi /(n+2)=\pi / \rho(A)$, we find that the assumptions in Theorem 12 are quite typical for solutions $A(z)$ of $g^{\prime \prime}+P(z) g=0$. If $n$ is an odd integer, then $A(z)$ is certainly not an exponential polynomial.

Theorem 13 Let $P(z)=a_{n} z^{n}+\cdots+a_{0}$ be a polynomial with real coefficients and $a_{n}>0$. Define $A(z)=P\left(e^{z}\right)$. Then the number of zeros $n([0, r), f)$ of a nontrivial real solution $f$ of (1.1) on the interval $[0, r)$ satisfies $n([0, r), f) \geq$ $C e^{n r / 2}$ for some constant $C>0$.

Proof. Define $q(x)=\frac{a_{n}}{2} e^{n x}$ for $x \in[0, \infty)$. Then $q(x)$ and $q^{\prime}(x)$ are continuous with $q^{\prime}(x) q(x)^{-3 / 2} \rightarrow 0$ as $x \rightarrow \infty$. Using either [9, Corollary 5.3] or [12, Theorem 9.5.1], we conclude the following: The number of zeros $n_{0}([0, x), y)$ of a non-trivial solution $y$ of $y^{\prime \prime}+q(x) y=0$ on the interval $[0, x)$ for large $x$ satisfies

$$
\begin{aligned}
n_{0}([0, x), y) & \sim \frac{1}{\pi} \int_{0}^{x} q(t)^{1 / 2} d t \geq \frac{1}{\pi} \int_{x-1}^{x} q(t)^{1 / 2} d t \\
& =\frac{\sqrt{2 a_{n}}}{n \pi}\left(1-e^{-n / 2}\right) e^{n x / 2} \geq \frac{\sqrt{2 a_{n}} \cdot 3}{10 n \pi} e^{n x / 2} .
\end{aligned}
$$

On the other hand, $A(z)$ is real-valued on the real axis, and $A(x) \geq q(x)$ for $x \geq r_{0}$. Thus all solutions of (1.1) are constant multiples of real entire functions. By the standard Sturm comparison theorem, any real solution $f$ vanishes at least once between any two zeros of $y$. Hence the magnitude of $n([0, x), f)$ is at least that of $n_{0}([0, x), y)$. This yields the assertion.

Example 12 If $P(z)=-z^{2}-z$, then (1.1) with $A(z)=P\left(e^{z}\right)$ has a zerofree solution $f(z)=\exp \left(e^{z}\right)$. Hence the assumption $a_{n}>0$ in Theorem 13 is essential.

Suppose that $A(z)$ is an exponential polynomial, and $\theta^{*}$ is such that $h_{A}\left(\theta^{*}\right)=\max _{\theta} h_{A}(\theta)(>0)$. We prove that most solutions of (1.1) have an infinite exponent of convergence in an arbitrarily small sector

$$
S\left(\theta^{*}, \varepsilon\right)=\left\{z \in \mathbb{C}:\left|\arg (z)-\theta^{*}\right|<\varepsilon\right\} .
$$

This is in contrast to the situation in Theorem [12. 
Theorem 14 Let $A(z)$ be an exponential polynomial of order $n$, let $\theta^{*} \in \mathbb{R}$ be such that $h_{A}\left(\theta^{*}\right)=\max _{\theta} h_{A}(\theta)$, and let $\varepsilon \in(0, \pi / 2 n)$. Moreover, suppose that (1.1) has a solution base $\left\{f_{1}, f_{2}\right\}$, and let $f_{3}$ be any linear combination of $f_{1}, f_{2}$ but not of the form $c f_{j}$. Then

$$
\max _{j=1,2,3}\left\{\lambda_{2}\left(S\left(\theta^{*}, \varepsilon\right), f_{j}\right)\right\}=n,
$$

where $\lambda_{2}\left(S\left(\theta^{*}, \varepsilon\right), f_{j}\right)$ is the hyper-exponent of convergence of the zeros of $f_{j}$ in the sector $S\left(\theta^{*}, \varepsilon\right)$.

Proof. For simplicity, we may suppose that $f_{3}=f_{1}-f_{2}$. Let $F=f_{1} / f_{2}$. Then the zeros, poles and 1-points of $F$ correspond to the zeros of $f_{1}, f_{2}, f_{3}$, respectively. Moreover, $2 A(z)=S_{F}(z)$, where

$$
S_{F}(z)=\frac{F^{\prime \prime \prime}(z)}{F^{\prime}(z)}-\frac{3}{2}\left(\frac{F^{\prime \prime}(z)}{F^{\prime}(z)}\right)^{2}
$$

is the Schwarzian derivative of $F$. For brevity, we indicate the angular domain $S\left(\theta^{*}, \varepsilon\right)$ by subindex $S$, and denote the angular Tsuji characteristic by $T_{S}(r, g)=m_{S}(r, g)+N_{S}(r, g)$, where $g$ is any meromorphic function. See [26, p. 58] for the definitions of these functions, but with different notation.

Recall from [17, p. 56] that

$$
h_{A}(\theta) \geq h_{A}\left(\theta^{*}\right) \cos \left(n\left(\theta-\theta^{*}\right)\right)
$$

for $\left|\theta-\theta^{*}\right| \leq \pi / n$. Hence, for our choice of $\varepsilon \in(0, \pi / 2 n)$, we have $h_{A}(\theta)>0$ for all $\theta \in\left[\theta^{*}-\varepsilon, \theta^{*}+\varepsilon\right]$. Since $A(z)$ grows fast in the sector $S\left(\theta^{*}, \varepsilon\right)$, we conclude by the angular second main theorem [26, p. 59] and by the angular lemma on the logarithmic derivative [26, pp. 85-86], that

$$
\begin{aligned}
C r^{n} \leq & m_{S}(r, A) \leq m_{S}\left(r, \frac{F^{\prime \prime \prime}}{F^{\prime}}\right)+2 m_{S}\left(r, \frac{F^{\prime \prime}}{F^{\prime}}\right)+O(1) \\
= & O\left(\log ^{+} T_{S}(2 r, F)\right)+O(\log r) \\
\leq & \log ^{+} N_{S}(2 r, \infty, F)+\log ^{+} N_{S}(2 r, 0, F)+\log ^{+} N_{S}(2 r, 1, F) \\
& +O\left(\log ^{+} \log ^{+} T_{S}(2 r, F)\right)+O(\log r) \\
\leq & 3 \max _{j=1,2,3}\left\{\log ^{+} N_{S}\left(2 r, 0, f_{j}\right)\right\}+O\left(\log ^{+} \log ^{+} T_{S}(2 r, F)\right)+O(\log r)
\end{aligned}
$$

outside of a possible exceptional set of $r$-values of finite linear measure. Since both sides of this inequality are increasing functions, we may avoid this exceptional set via [16, Lemma 1.1.1]. This proves $\max _{j=1,2,3}\left\{\lambda_{2}\left(S\left(\theta^{*}, \varepsilon\right), f_{j}\right)\right\} \geq n$. Since the inequalities

$$
\max _{j=1,2,3}\left\{\lambda_{2}\left(S\left(\theta^{*}, \varepsilon\right), f_{j}\right)\right\} \leq \max _{j=1,2,3}\left\{\lambda_{2}\left(f_{j}\right)\right\} \leq \max _{j=1,2,3}\left\{\rho_{2}\left(f_{j}\right)\right\} \leq n
$$


are clear, the assertion follows.

Acknowledgement. The authors want to thank James Langley for helpful discussions. In particular, Theorem 10 is essentially due to Langley.

\section{References}

[1] Bank S. and I. Laine, On the oscillation theory of $f^{\prime \prime}+A f=0$ where $A$ is entire, Trans. Amer. Math. Soc. 273 (1982), no. 1, 351-363.

[2] Bank S., I. Laine and J. Langley, On the frequency of zeros of solutions of second order linear differential equations, Resultate Math. 10 (1986), 8-24.

[3] Bank S., I. Laine and J. Langley, Oscillation results for solutions of linear differential equations in the complex domain, Resultate Math. 16 (1989), 3-15.

[4] Bergweiler W. and A. Eremenko, On the Bank-Laine conjecture, J. Eur. Math. Soc. (JEMS) 19 (2017), no. 6, 1899-1909.

[5] Bergweiler W. and A. Eremenko, Quasiconformal surgery and linear differential equations, arXiv:1510.05731

[6] Frank G. and W. Hennekemper, Einige Ergebnisse über die Werteverteilung meromorpher Funktionen und ihrer Ableitungen, Resultate Math. 4 (1981), 39-54.

[7] Frank G., X. Hua and R. Vaillancourt, Meromorphic functions sharing the same zeros and poles, Canad. J. Math. 56 (2004), 1190-1227.

[8] Gundersen G., Estimates for the logarithmic derivative of a meromorphic function, plus similar estimates, J. London Math. Soc. 37 (1988), no. 1, 88-104.

[9] Hartman P., Ordinary Differential Equations. 2nd ed., Siam, Philadelphia, 2002.

[10] Hayman W., Meromorphic Functions. Oxford Mathematical Monographs, Clarendon Press, Oxford, 1964.

[11] Heittokangas J., I. Laine, K. Tohge and Z.-T. Wen, Completely regular growth solutions of second order complex linear differential equations, Ann. Acad. Sci. Fenn. Math. 40 (2015), 985-1003.

[12] Hille E., Lectures on Ordinary Differential Equations. Addison-Wesley Publ. Co., Reading, Mass.-London-Don Mills, Ont., 1969.

[13] Hinkkanen A. and J. Rossi, Schwarzian derivatives and zeros of solutions to second order linear differential equations, Proc. Amer. Math. Soc. 113 (1991), no. 3, 741-746.

[14] Ishizaki K., An oscillation result for a certain linear differential equation of second order, Hokkaido Math. J. 26 (1997), no. 2, 421-434.

[15] Ishizaki K. and K. Tohge, On the complex oscillation of some linear differential equations, J. Math. Anal. Appl. 206 (1997), no. 2, 503-517.

[16] Laine I., Nevanlinna Theory and Complex Differential Equations. Walter de Gruyter, Berlin-New York, 1993. 
[17] Levin B. Ja., Distribution of Zeros of Entire Functions. Translated from the Russian by R. P. Boas, J. M. Danskin, F. M. Goodspeed, J. Korevaar, A. L. Shields and H. P. Thielman. Revised edition. Translations of Mathematical Monographs, 5. American Mathematical Society, Providence, R.I., 1980.

[18] Nehari Z., Conformal Mapping. Reprinting of the 1952 edition. Dover Publications, Inc., New York, 1975.

[19] Schwarz B., Complex nonoscillation theorems and criteria of univalence, Trans. Amer. Math. Soc. 80 (1955), 159-186.

[20] Steinbart E., On the location of zeros of solutions to $w^{\prime \prime}+A w=0$, Funkcial. Ekvac. 35 (1992), no. 2, 223-254.

[21] Steinmetz N., Zur Wertverteilung von Exponentialpolynomen, Manuscripta Math. 26 (1978/79), no. 1-2, 155-167.

[22] Steinmetz N., Zur Wertverteilung der Quotienten von Exponentialpolynomen, Arch. Math. (Basel) 35 (1980), no. 5, 461-470.

[23] Tsuji M., Potential Theory in Modern Function Theory. Reprinting of the 1959 original. Chelsea Publishing Co., New York, 1975.

[24] Tu J. and X. D. Yang, On the zeros of solutions of a class of second order linear differential equations, Kodai Math. J. 33 (2010), 251-266.

[25] Yang C.-C. and H.-X. Yi, Uniqueness Theory of Meromorphic Functions. Mathematics and its Applications, 557. Kluwer Academic Publishers Group, Dordrecht, 2003.

[26] Zheng J., Value Distribution of Meromorphic Functions. Tsinghua University Press, Beijing; Springer, Heidelberg, 2010.

Janne Heittokangas

TAIYUAN UNIVERSITY OF TECHNOLOGY

FACULTy OF MATHEMATICS

Yingze West Street, No. 79, Taiyuan 030024, China

email: janne.heittokangas@uef .fi

Ilpo Laine 63 Janne Heittokangas

UNIVERSITY OF EASTERN FINLAND

Department of Physics and Mathematics

P.O. Box 111, 80101 Joensuu, Finland

email:ilpo.laine@uef.fi

Katsuya Ishizaki

The Open UNIVERSITY OF JAPAN

2-11 WaKaba MiHama-KU Chiba

261-8586, JAPAN

email:ishizaki@ouj.ac.jp

Kazuya Tohge

KANAZAWA UNIVERSITY

College of Science and Engineering

KAKUMA-MACHI, KANAZAWA 920-1192, JAPAN

email:tohge@se.kanazawa-u.ac.jp 